\section{Stéphane Richard Sylviane Olschwang Dominique Chauveau François Resche}

\title{
La maladie de von Hippel-Lindau
}

La maladie de von Hippel-Lindau est une maladie génétique rare, transmise sur le mode autosomique dominant, comportant le développement de tumeurs variées dont les plus caractéristiques affectent le cervelet, la moelle épinière, la rétine (hémangioblastomes), les reins (kystes et cancers), les surrénales (phéochromocytomes) et le pancréas (kystes et tumeurs). Elle est due à l'altération d'un gène unique, situé en 3 p25-26, codant pour une protéine dont on ne connaît pas encore la fonction. L'identification du gène responsable de la maladie a été l'occasion d'un grand progrès clinique car elle permet de conduire l'analyse génétique dans les familles atteintes, de surveiller les sujets à risque et de dépister et traiter les lésions à leur début.

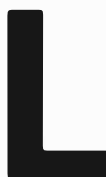

'affection à laquelle Eugen von Hippel, ophtalmologiste allemand (1895) [1], et Arvid Lindau, pathologiste suédois (1926) [2], ont laissé leurs noms est caractérisée par le développement de tumeurs affectant le système nerveux central et la rétine (hémangioblastomes), les reins (kystes et cancers), les surrénales (phéochromocytomes) et le pancréas (kystes ou tumeurs) (figure 1) [3, 4]. Transmise sur le mode autosomique dominant avec une forte pénétrance $(95 \%$ à 60 ans), son incidence est de $1 / 36000$ à $1 / 40000$ et les manifestations cliniques apparaissent habituellement entre 18 et 30 ans $\left(m / s n^{\circ} 6\right.$, vol. $7, p$. $632[3,4])$.

Si l'on excepte Melmon et Rosen en 1964 [5], il a fallu attendre la localisation du gène dont les altérations sont responsables de la maladie en 1988 , pour que les travaux consacrés à l'affection connaissent un nouvel essor et révèlent l'importance de lésions viscérales longtemps considérées comme de simples curiosités [3].
Fascinante pour le chercheur, la présentation protéiforme de la maladie de von Hippel-Lindau (VHL) est très déroutante pour le clinicien. Il en résulte des retards diagnostiques aux conséquences dramatiques alors que la plupart des lésions sont accessibles à un traitement précoce. L'identification récente du gène dont les altérations sont responsables de la maladie et la description prochaine du spectre de ses mutations constitutionnelles devraient permettre une meilleure compréhension de la pathogénie de cette phacomatose méconnue.

\section{Clinique}

Une parfaite connaissance des multiples manifestations de la maladie, une enquête génétique rigoureuse et des investigations cliniques et paracliniques complètes sont les clés du diagnostic.

Les critères suivants, proches de ceux définis par Melmon et Rosen, sont communément admis [3-6]: le dia-

S. Richard. 
gnostic de maladie de VHL doit être porté chez tout patient porteur d'au moins deux hémangioblastomes ou d'un hémangioblastome et d'une lésion viscérale (phéochromocytome, kystes rénaux multiples, cancer du rein avant l'âge de 45 ans, kystes ou tumeurs pancréatiques). Certaines équipes y incluent encore la présence de cystadénomes de l'épididyme sous réserve d'une confirmation anatomo-pathologique [7]. En présence d'une histoire familiale démonstrative, une seule des lésions citées suffit au diagnostic.

\section{L'hémangioblastome du système ner- veux central}

\section{RÉFÉRENCES}

1. Von Hippel E. Ueber eine sehr seltene Erkrankung der Netzhaut. Klinische Beobachtungen. Von (iraefe Arch Ophthalmol $19(04 ; 59: 83-106$.

2. Lindau A. Studien über Kleinhirnzysten. Bau, Pathogenese und Beziehungen zur Angiomatosis retinae. Acta Pathol Microbiol Scand 1926 ; S I : 1-128.

3. Lamiell JM, Salazar FG, Hsia YE. Von Hippel-Lindau disease affecting 43 members of a single kindred. Medicine $1989 ; 68$ : $1-29$.

4. Maher ER, Yates JRW, Harries R, Benjamin C, Moore AT, Ferguson-Smith MA. Clinical features and natural history of von Hippel-Lindau disease. Q/ Med $1990 ; 283$ : 1151-63.

5. Melmon KL, Rosen SW. Lindau's disease: review of the literature and study of a large kindred. Am J Med 1964 ; 36 : 595-617.

6. Resche F, Moisan JP, Mantoura J, et al. Hemangioblastomas, hemangioblastomatosis and von Hippel-Lindau disease. Adv Techn Stand Neurosurg $1993 ; 2(): 197-3() 3$.

7. Filling-Katz MR, Choyke PL, Oldfield E, et al. Central nervous system involvement in von Hippel-Lindau disease. Neurology 1991 ; $41: 41-6$.

8. Neumann HPH, Eggert HR, Scheremer R, et al. Central nervous system lesions in von Hippel-Lindau syndrome. I Neurol Neurosurg Psychiatry 1992 ; 55 : 898-901

9. Frank TS, Trojanowski JQ, Roberts SA, Brooks I . A detailed immunohistochemical analysis of cerebellar hemangioblastoma: an undifferentiated mesenchymal tumor. Mod Pathol 1989 ; 2: 638-51.

10. Maher ER, Moore AT. Von Hippel-Lindau disease. $\mathrm{Br} /$ Ophthalmol $1992 ; 76$ : 7435 .

11. Choyke PL, Glenn GM, Walther McCM, et al. The natural history of renal lesions in von Hippel-Lindau disease. Am / Roentgen
Lésion vasculaire bénigne, constituée d'un abondant réseau capillaire dont les mailles renferment des éléments tumoraux riches en lipides (cellules stromales), l'hémangioblastome (ou "angioréticulome») est la manifestation la plus constante de l'affection (60\% à $80 \%$ des cas, première manifestation 1 fois sur 2) [6, 7]. L'hémangioblastome, qui représente $2 \%$ à $3 \%$ de l'ensemble des tumeurs cérébrales, se développe avec prédilection au niveau de la fosse postérieure (cermoelle épinière [6]. Au cours de la maladie de VHL, les tumeurs sont fréquemment multiples ("hémangioblastomatose") (figure 2) et se révèlent en moyenne quinze ans plus tôt que les hémangioblastomes sporadiques $[6,8]$. La localisation infratentorielle (60\% des cas) est responsable d'une hypertension intracrânienne et d'un syndrome cérébelleux. La classique polyglobulie secondaire serait due à la sécrétion d'érythropoïétine par la tumeur. Les localisations rachidiennes, souvent asymptomatiques, affectent $30 \%$ à $40 \%$ des patients [7] : la jonction cervico-médullaire et le cône terminal en sont les deux régions d'élection. Les formes sus-tentorielles sont exceptionnelles.

L'histogenèse de l'hémangioblastome reste obscure malgré de nombreux travaux ultrastructuraux et immunocytochimiques. Les cellules stromales présentent de manière constante une positivité vis-à-vis des anticorps dirigés contre la vimentine, traduisant une nature "mésenchymateuse indifférenciée" (pour revue, $[6,9])$. velet, bulbe), plus rarement de la

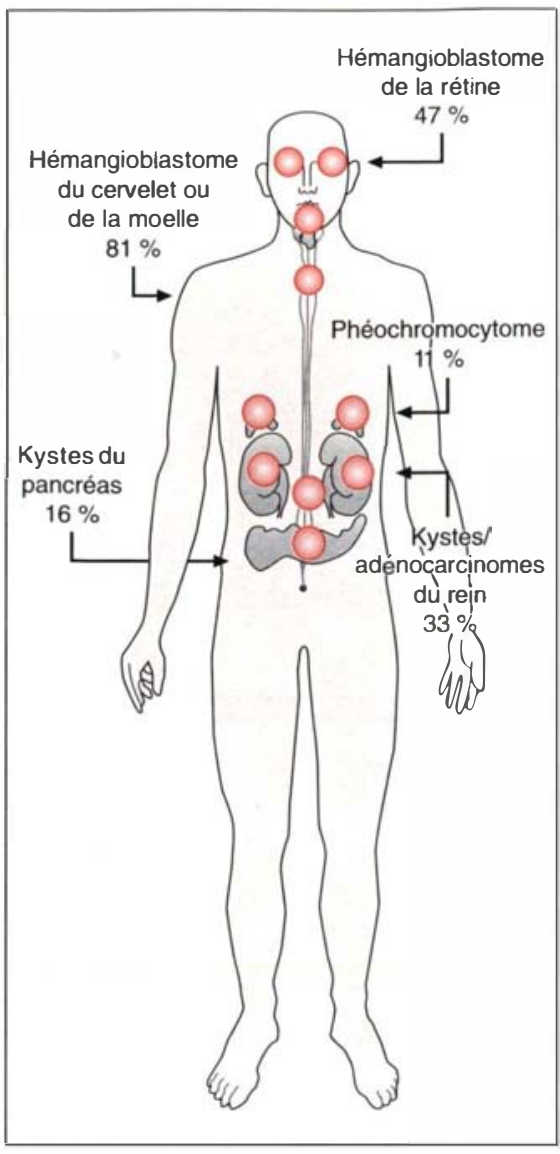

Figure 1. Principales lésions de la maladie de von Hippel-Lindau. Fréquence des différentes atteintes dans la série française de 380 observations.

L'hémangioblastome rétinien, habituellement multiple et bilatéral ( «angiomatose rétinienne»), affecte un malade sur deux et révèle la maladie dans $30 \%$ des cas. Il s'agit probablement de la lésion la plus évocatrice de l'affection, quasi pathognomonique [10], l'existence d'hémangioblastomes rétiniens en dehors de la maladie de VHL apparaissant rarissime [3]. Ils se situent préférentiellement à la périphérie de la rétine mais des localisations juxta-papillaires sont également observées. Leur évolution naturelle, en l'absence de traitement, se complique de décollement rétinien et de glaucome aboutissant à la destruction oculaire.

\section{Une atteinte viscérale au premier plan}

Bien que parfaitement comprises dès 1926 par Lindau [2], les manifesta- 


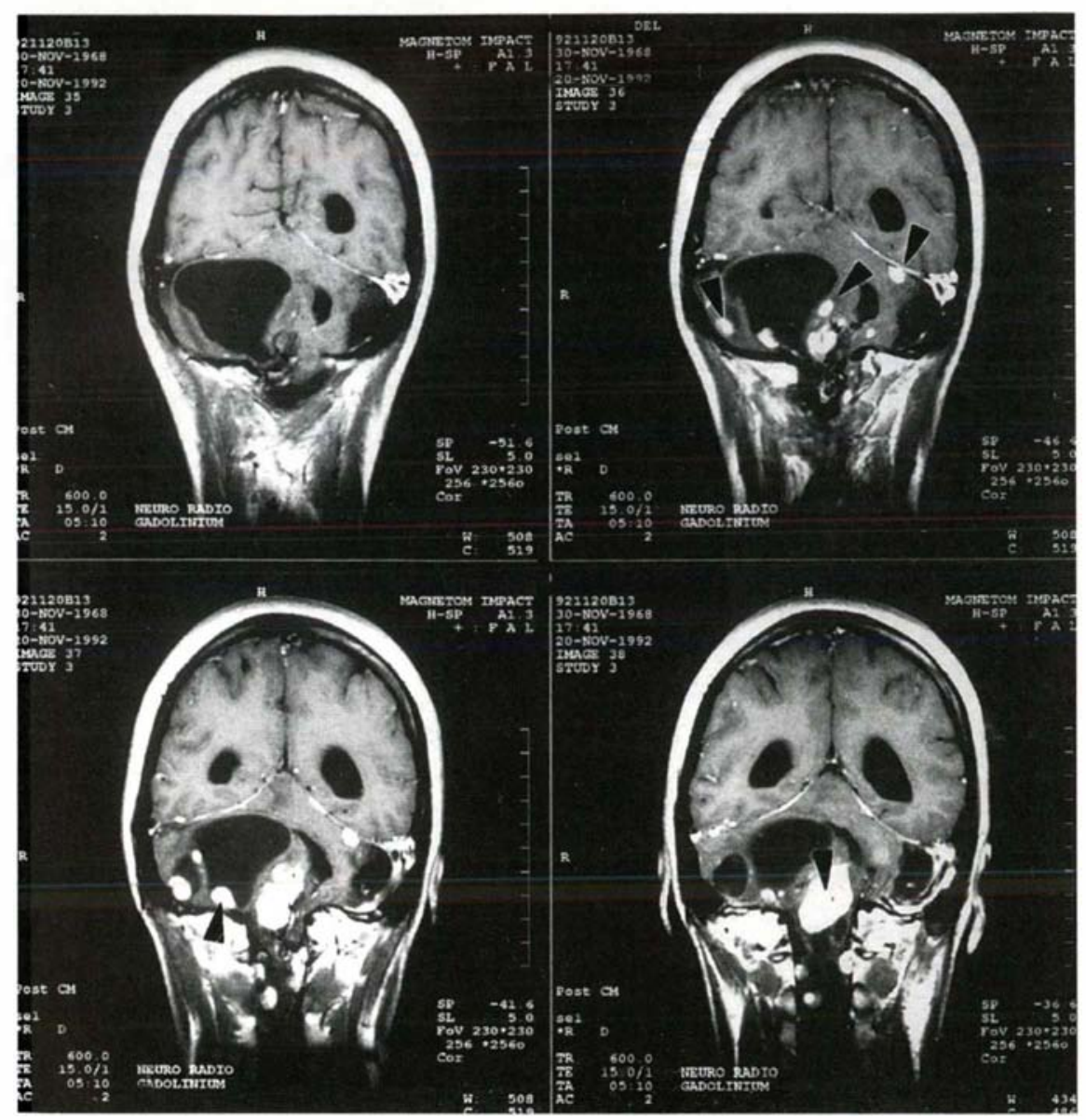

Figure 2. Hémangioblastomes multiples (pointes de flèches), le plus souvent kystiques, de la fosse postérieure chez une patiente de 19 ans. Imagerie par résonance magnétique (IRM) avec injection de gadolinium.

tions viscérales de la maladie demeurent encore souvent méconnues. La fréquence des lésions rénales est ainsi directement proportionnelle à l'attention accordée à leur recherche systématique (de $30 \%$ à $60 \%$ selon les séries) [11, 12]. Le cancer du rein, qui présente toujours histologiquement l'aspect d'un adénocarcinome à cellules claires, fait toute la gravité de la maladie. Longtemps asymptomatique, son jeune âge de découverte (60\% avant 40 ans), une fréquente apparence kystique et son caractère multifocal et bilatéral le distinguent des cancers rénaux "sporadiques" (figure 3) [12]. Des kystes simples, multiples et corticaux, s'observent également.

En dépit du bas grade nucléaire de la plupart des tumeurs observées et d'une évolution longtemps locale, la fréquence des métastases ne semble pas différente de celle des cancers du rein sporadiques $[3,4]$.

L'histogenèse du cancer du rein demeure mystérieuse. Sur les pièces opératoires, une transition progressive des kystes simples aux carcinomes est habituelle en passant par tous les intermédiaires de kystes "atypiques", et la présence de foyers carcinomateux intra-kystiques s'observe déjà sur des lésions de quelques millimètres [13]. Des arguments immunohistochimiques indiquent toutefois que les kystes bénins naîtraient surtout des tubes contournés distaux alors que les cancers et les kystes atypiques expriment des marqueurs proximaux [14]. Des données radiologiques sont également en faveur d'une distinction entre kystes simples d'une part, kystes atypiques et cancers d'autre part. Choyke et al. [11] ont suivi par tomodensitométrie abdominale périodique vingt-huit patients porteurs de lésions rénales de la maladie de VHL sur une période allant de 1 à 12 ans (moyenne 2,4 ans). La majorité $(71 \%)$ des lésions kystiques pures est restée inchangée tandis que les kystes atypiques subissaient un accroissement régulier de la composante charnue et que les lésions tumorales solides (cancers classiques) augmentaient rapidement de volume (temps de doublement: dix mois).

Le phéochromocytome, fréquemment bilatéral, survient dans $10 \%$ à $19 \%$ des cas de maladie de VHL [3, 12]. Ia triade classique céphaléespalpitations-sueurs est rare, la symptomatologie souvent fruste (HTA isolée), les dosages des catécholamines et de leurs métabolites parfois normaux. La découverte du phéochromocytome peut être fortuite, sur une pièce de néphrectomie pour cancer du rein. Le phéochromocytome est habituellement surrénalien et exceptionnellement malin au cours de la maladie de VHL.

L’importance clinique et génétique du phéochromocytome réside dans son observation très préférentielle dans certaines familles [15, 16], son caractère souvent révélateur [17] et le risque latent d'hypertension paroxystique.

L'atteinte pancréatique, qui affecte de $15 \%$ à $38 \%$ des patients, consiste essentiellement en une polykystose asymptomatique. D'authentiques tumeurs exocrines (cystadénomes, carcinomes) ou endocrines (nésidioblastomes) peuvent cependant survenir [18].

\section{Les lésions mineures}

Des cystadénomes épididymaires sont observés chez $15 \%$ des malades. Ils sont encore souvent considérés comme l'un des signes cardinaux de l'affection malgré leur fréquence dans la population générale [3]. Seizinger $e t$ al. [19] ont pu démontrer, par des études de liaison génétique, qu'il ne s'agissait pas d'un critère fiable de diagnostic, leur présence, même bilatérale chez un sujet à risque, pouvant être parfaitement indépendante de la maladie.

Parmi les quelques quinze autres atteintes différentes qui ont été dé- 


\section{RÉFÉRENCES \\ 12. Richard S, Chauveau D, Chrétien Y, et al. Renal lesions and pheochromocytoma in von Hippel-Lindau disease. Adv Nephrol $1993 ; 23: 1-27$.}

13. Solomon D, Schwartz A. Renal patholo13. Solomon D, Schwartz A. Renal patholo-
gy in von Hippel-Lindau disease. Hum Pathol $1988 ; 19: 1072-9$.

14. Kragel PJ, Walther MC:M, Pestaner JP, et al. Simple renal cysts, atypical renal cysts, and renal cell carcinoma in von Hippel-Lindau disease: a lectin and immunohistochemical study in six patients. Mod Pathol 1991 ; $4: 21()-4$.

15. Atuk NO, McDonald T, Wood T, et al. Familial pheochromocytoma, hypercalcemia, and von Hippel-Lindau disease. Medicine $1979 ; 58: 209-18$.

16. Creen JS, Bowmer MI, Johnson CiJ. Von Hippel-Lindau disease in a Newfoundland kindred. Can Med Assoc J 1986 ; 134 : 133-46.

17. Richard S, Beigelman C, Duclos JM, et al. Pheochromocytoma as the first manifestation of von Hippel-Lindau disease. Surgery 1995 (sous presse).

18. Neumann HPH, Dinkel E, Brambs H, el al. Pancreatic lesions in the von Hippel-Lindau syndrome. Gastroenterology 1991; 101: 465-71.

19. Seizinger BR, Smith D, Filling-Katz D, el al. Genetic flanking markers refine diagnostic criteria and provide new insights into the genetics of von Hippel-Lindau disease. Proc Natl Acad Sci USA 1991 ; 88 : 2864-8.

20. Neumann HPH, Wiestler OD. Clustering of features of von Hippel-Lindau syndrome: evidence for a complex genetic locus. Lancet $1991 ; 337: 1052-4$.

21. Poe I)S, Tarlov EC; Thomas ('B, Kveton JF. Aggressive papillary tumors of temporal bone. Otolaryngol Head Neck Surg 1993; 108 : 8()$-6$

22. (jlenn (;M, Daniel LN, Choyke P, et al. Von Hippel-Lindau (VHL) disease: distinct phenotypes suggest more than one mutant allele at the VHL locus. Hum (ienet 1991 87: $207-10$.

23. Maher ER, Iselius I, Yates JRW', et al. Von Hippel-Lindau disease : a genetic stu-

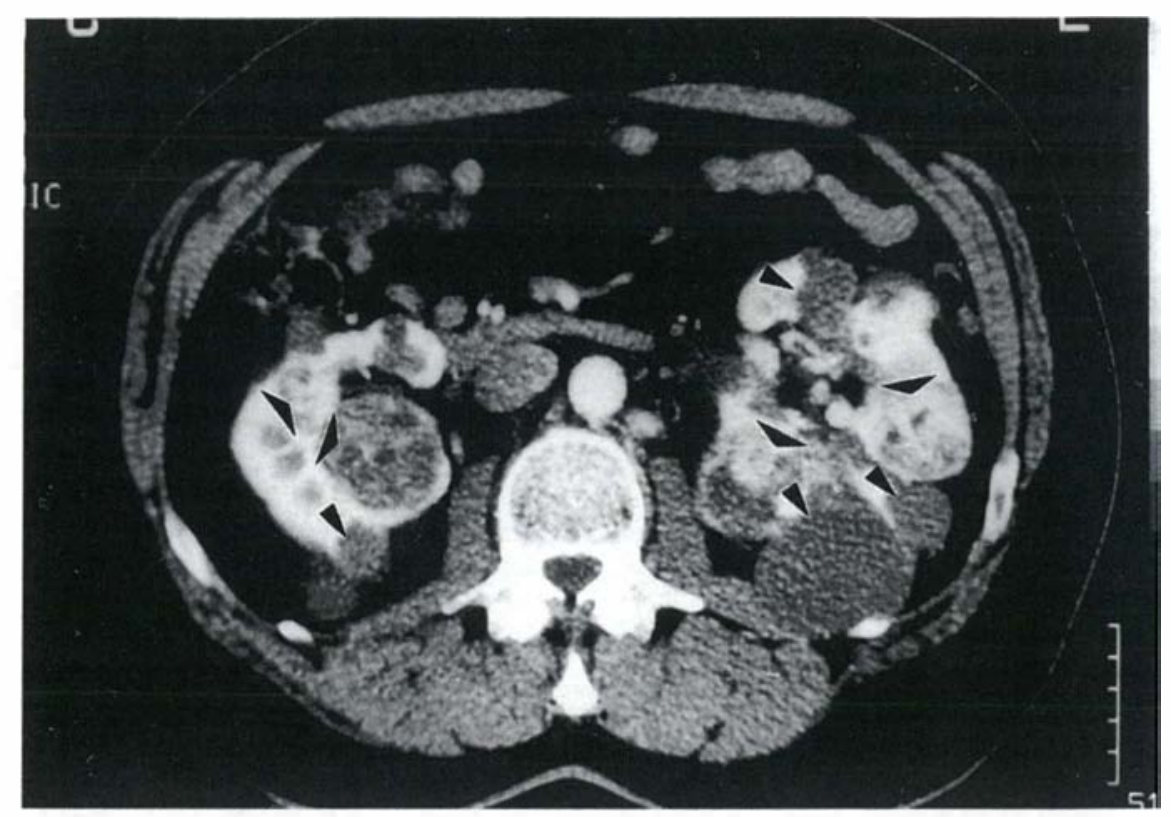

Figure 3. Lésions rénales bilatérales et multifocales chez une patiente de 30 ans, rendant impossible une chirurgie conservatrice : tumeurs (flèches aiguës) et kystes (flèches plates). Tomodensitométrie (TDM) abdominale après injection de produit de contraste.

crites en association avec la maladie [20], et dont la plupart correspondent vraisemblablement à des associations fortuites, une lésion mérite cependant d'être individualisée. Les tumeurs glandulaires de l'apex pétreux (papillomes ou carcinomes du sac endolymphatique) sont des lésions ORL rares dont l'association avec la maladie de VHL ( 8 fois sur 40 cas décrits) semble en effet plus qu'une coïncidence [21].

Enfin, contrairement aux autres phacomatoses, il n'y a pas de signe cutané au cours de la maladie de VHL.

Une grande hétérogénéité phénotypique

A l'intérieur d'une même famille, le siège et le nombre des lésions pathologiques, l'âge et le type de la première manifestation et la sévérité de l'évolution varient grandement d'un sujet à l'autre [18,22]. Le nombre des lésions découvertes est avant tout fonction de la qualité du suivi des patients (explorations complètes, vérification nécropsique en cas de décès). En Angleterre, des hémangioblastomes rétiniens ont ainsi été trouvés chez $60 \%$ de sujets atteints n'ayant jamais eu de bilan ophtalmologique auparavant [10]. Choyke et al., qui se sont particulièrement intéressés à l'atteinte rénale, en ont découvert une chez $63 \%$ des malades explorés [4]. La série française, à point de départ neurochirurgical, comporte $80 \%$ d'hémangioblastomes du système nerveux central [12]. Une grande hétérogénéité se retrouve cependant dans des familles parfaitement explorées et prises en charge (figure 4).

Le phéochromocytome représente un problème très particulier qui doit être individualisé. Il s'observe préférentiellement dans certaines familles de malades où sa fréquence est alors très élevée, jusqu'à $90 \%$ des sujets atteints (familles dites "à phéochromocytome») $[12,15,16]$.

\section{Génétique}

\section{Épidémiologie}

I a maladie de VHL est une maladie héréditaire à transmission autosomique dominante. La pénétrance augmente avec l'âge et la plupart des sujets porteurs du gène altéré auront 


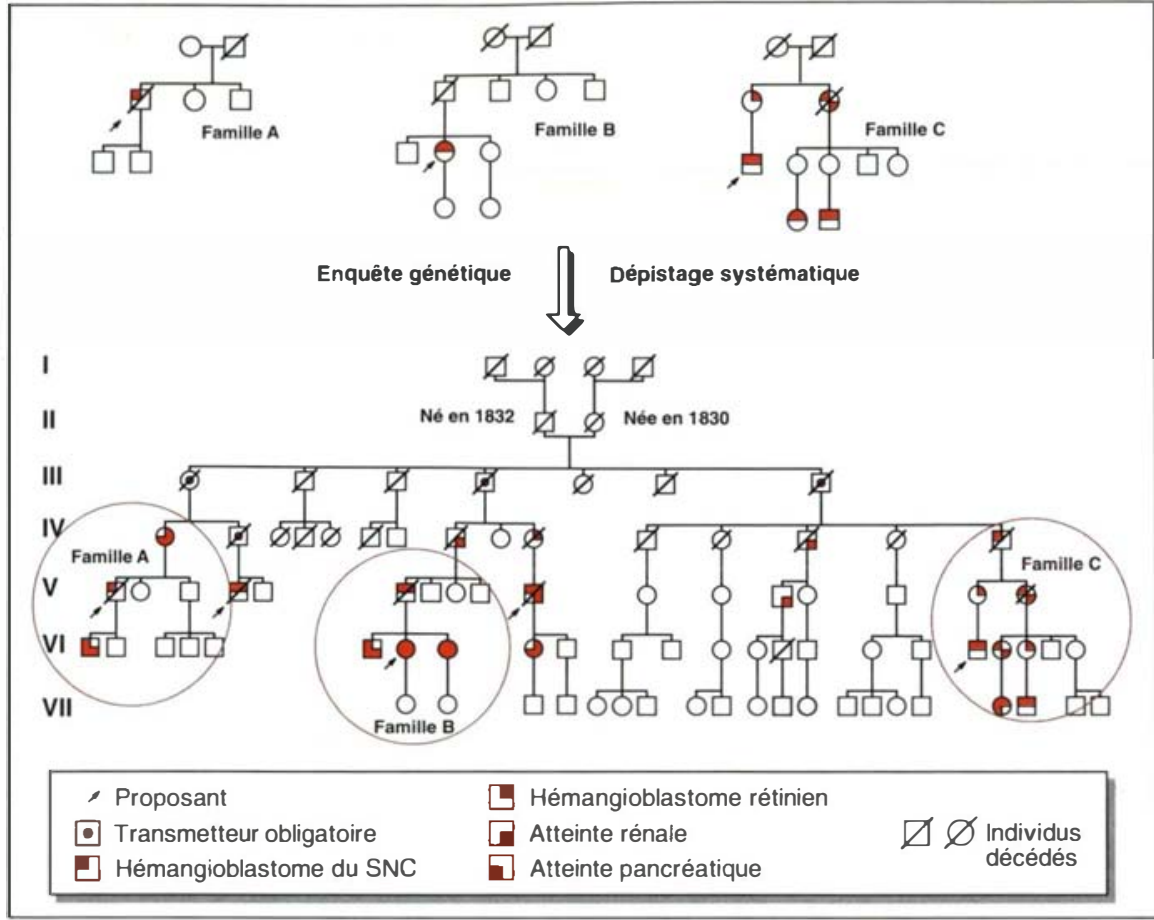

Figure 4. Reconstitution de la famille " 8 " par généalogie ascendante à partir de 3 malades indépendants. Cette famille comporte 21 malades connus et 4 transmetteurs obligatoires sur 6 générations.

développé une tumeur avant l'âge de 30 ans.

Affection ubiquitaire, la maladie de VHL a été particulièrement étudiée dans l'est de l'Angleterre, où des registres régionaux ont été établis. Ils ont permis d'estimer l'incidence de la maladie à un nouveau cas pour 36000 naissances et la prévalence à un sujet atteint pour 53000 habitants [23].

En France, l'étude débutée en 1990 a permis de recenser plus de 380 observations et 85 familles. Les études de génétique formelle systématiques ont révélé quelques berceaux principaux (figure 5). Les foyers les plus importants de la maladie de VHL se situent dans le Centre-Ouest et notamment dans le Cher à proximité de Sancerre (figure 4).

La fréquence des néo-mutations, estimée d'après le nombre de cas apparemment sporadiques, semble faible dans la maladie de VHL. La proportion de tels cas est en diminution constante en France (13\% actuellement) et tient sans doute au caractère systématique des enquêtes génétiques qui bien souvent permettent de rattacher des patients "isolés" à $m / s n^{\circ} l$, vol. 11 , janvier 95 térozygotie ciblées sur le bras court du chromosome 3 ont progressivement permis de déterminer la région minimale altérée (figure 6) [29, 30].

Le gène VHL: localisation génomique

L'origine de nombreuses maladies héréditaires a été précisée par la génétique moléculaire. Une des méthodes utilisées est basée sur la variabilité génétique existant dans l'espèce humaine; de nombreux loci polymorphes peuvent être caractérisés grâce à des "sondes" qui détectent, sur l'ADN génomique, des différences entre les individus d'une population. Dans un ensemble de familles présentant une même pathologie héréditaire, l'une de ces "sondes" peut mettre en évidence une liaison génétique entre le locus correspondant et la maladie, liaison qui traduit la proximité du locus avec l'altération génétique responsable.

La première liaison génétique entre le gène $V H I$, et un locus polymorphe, situé en 3p25, a été mise en évidence par Seizinger et al. en 1988 [31]. Quelques rares événements de recombinaison méiotique entre le locus morbide et le proto-oncogène $R A F 1$ ont permis d'éliminer la responsabilité de ce dernier, qui pouvait être un gène candidat pour la maladie de VHL. En outre, les arguments étaient forts pour penser que le gène VHI avait un rôle suppresseur de tumeur, étant donné les multiples observations, dans les tumeurs associées à la maladie de VHL, de pertes chromosomiques acquises dans cette région du génome, affectant toujours l'allèle issu du parent sain [30], ainsi que les différences significatives d'âges de révélation entre les tumeurs de la maladie de VHL et les tumeurs sporadiques (cancer rénal, hémangioblastome) [32].

\section{Identification}

Aucune étude n'ayant montré d'hétérogénéité génétique, malgré l'expression très variable de la maladie, l'existence d'un seul gène sur le chromosome 3 , dont les altérations seraient responsables des différentes formes cliniques, a été évoquée. Plusieurs cartes génétiques de la région 3p25-p26 ont été établies avec diffé- 


\section{RÉFÉRENCES}

24. King CR, Schimke RN, Arthur T, Davoren B, Collins D. Proximal $3 p$ deletion in renal carcinoma cells from a patient with von Hippel-Lindau disease. Cancer Genet Cytogenet 1987 ; 27 : 345-8.

25. Decker HJH, Neumann HPH, Walter TA, Sandberg AA. 3p involvement in a renal cell carcinoma in von Hippel-Lindau syndrome. Region of tumor breakpoint clustering on $3 p$ ? Cancer Genet Cytogenet 1988; 33 : $59-65$

26. Kovacs G, Emanuel A, Neumann HPH, Kung HF. Cytogenetics of renal cell carcinomas associated with von Hippel-Lindau disease. Genes Chrom Cancer 1991 ; 3 : 256-62.

27. Kiechle-Schwarz M, Neumann HPH, Decker HJH, Dietrich C, Wullich B, Schempp $W$. Cytogenetic studies on three pheochromocytomas derived from patients with von Hippel-Lindau syndrome. Hum Genet $1989 ; 82: 127-30$

28. Boldog F, Arheden K, Imreh S, et al. Involvement of $3 p$ deletions in sporadic and hereditary forms of renal cell carcinoma. Genes Chrom Cancer 1991; 3 : 403-6.

29. Yamakawa $\mathrm{K}$, Morita R, Takahashi EI, Hori TA, Ishikawa I, Nakamura Y. A detailed deletion mapping of the short arm of chromosome 3 in sporadic renal cell carcinoma. Cancer Res $1991 ; 51$ : 4707-11.

30. Tory KH, Brauch $\mathrm{H}$, Linehan $\mathrm{M}$, et al. Specific genetic change in tumors associated with von Hippel-Lindau disease. I Natl Cancer Inst $1989 ; 81$ : 1097-101.

31. Seizinger BR, Rouleau GA, Ozelius LJ, et al. Von Hippel Lindau disease maps to the region of chromosome 3 associated with renal cell carcinoma. Nature 1988 ; 332 : 2689.

32. Maher ER, Yates JRW, Ferguson-Smith MA. Statistical analysis of the two stage mutation model in von Hippel-Lindau disease, and in sporadic cerebellar haemangioblastoma and renal cell carcinoma. $J$ Med Genet $1990 ; 27: 311-4$

33. Crossey PA, Maher ER, Jones MH, et al. Genetic linkage between von Hippel-Lindau disease and three microsatellite polymorphisms refines the localisation of the VHL locus. Hum Mol Genet 1993 ; 2 : 279-82.

34. Maher ER, Bentley E, Yates JRW, et al. Mapping of the von Hippel-Lindau disease locus to a small region of chromosome $3 p$ rentes séries de sondes de polymorphisme [20, 33-37]. Une cartographie physique à partir des loci les plus proches a été entreprise (figure6). Elle a permis d'éliminer un autre gène candidat de la région, $P M C A 2$, et a abouti au clonage du gène $V H I$. en mai $1993\left(\mathrm{~m} / \mathrm{s} n^{\circ} 8-9\right.$, vol. 9 , p. 988 $[38,39])$. Ce gène s'étend sur une longueur d'environ 50000 paires de bases et sa structure génomique n'est pas connue actuellement.

I.e gène VHI, est exprimé dans tous les tissus et à tous les stades du développement examinés. Cependant, deux formes d'ARN messager mûr ont été identifiées, longues de 6 et $6,5 \mathrm{~kb}$, qui diffèrent vraisemblablement par l'existence d'un site alternatif d'épissage, et dont l'expression n'est pas équivalente. Le transcrit court est absent du rein foetal, et le transcrit long, absent du cerveau fotal, alors que les deux coexistent dans tous les tissus adultes. Les transcrits mûrs comportent une longue séquence non traduite en 3' et les trois exons actuellement identifiés codent pour une protéine de 284 acides aminés. Cette dernière comporte une forte analogie de séquence avec une protéine de $\%$. bmucei, par la présence de 8 pentamères répétés en tandem, créant un domaine acide. Ce domaine évoque la possibilité d'une localisation membranaire de la protéine, et son éventuelle implication dans la transmission du signal ou l'adhérence cellulaire.

Des altérations, constitutionnelles d'une part, responsables de la maladie de VHL, et acquises de l'autre, dans des cancers du rein sporadiques, ont été rapportées, suggérant que le gène $V H I$, et le gène impliqué dans les cancers du rein sporadiques étaient identiques [38]. Au niveau somatique, des mutations ont été trouvées dans plus de la moitié des cancers du rein à cellules claires [40]. I.es altérations constitutionnelles peuvent être de deux types : un quart résulte de délétions interstitielles du bras court du chromosome 3, les remaniements génomiques étant détectés en utilisant la méthode de Southern après électrophorèse conventionnelle ou en champs pulsés (figure 6). Le plus fréquemment, il s'agit de mutations ponctuelles affectant la séquence codante du gène (une quarantaine déjà décrites) [41]. Leur conséquence prévisible est, dans la majorité des cas, la synthèse d'une protéine tronquée. Une association significative, entre une substitution non conservative de l'exon 3 (Arg $\rightarrow$ Trp ou Arg $\rightarrow$ Gln au codon 238) et l'existence de phéochromocytomes chez les sujets atteints des familles correspondantes, a été mise en évidence.

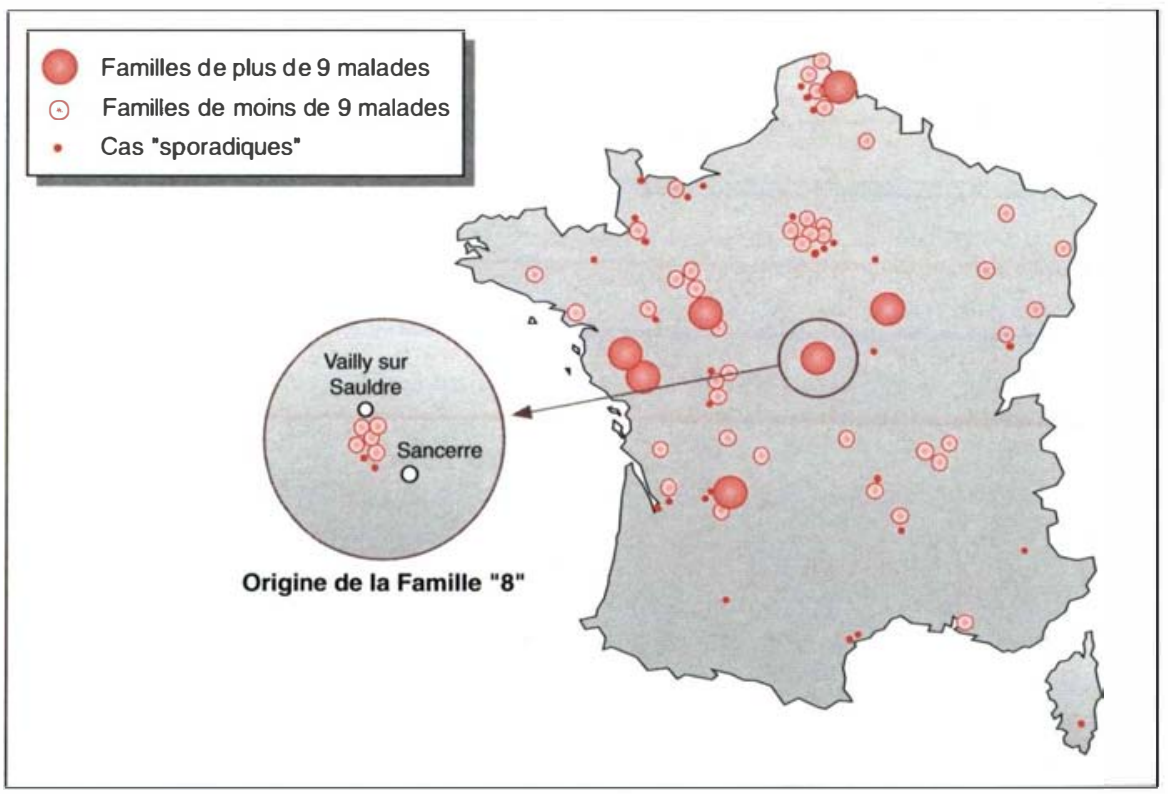

Figure 5. Origine géographique des familles françaises atteintes par la maladie de von Hippel-Lindau. Le principal foyer se situe dans le Cher, à proximité de Sancerre (famille " 8 "). 
Tableau I

PRISE EN CHARGE DES PATIENTS ET DE LEUR FAMILLE

\begin{tabular}{|c|c|c|c|c|c|c|}
\hline \multirow[t]{2}{*}{ Lésion } & \multirow{2}{*}{$\begin{array}{c}\text { Age de } \\
\text { révélation' } \\
\text { (ans) }\end{array}$} & \multirow[t]{2}{*}{ Dépistage } & \multicolumn{3}{|c|}{ Périodicité des examens } & \multirow[t]{2}{*}{ Traitement } \\
\hline & & & Début & Malades & A risque & \\
\hline $\begin{array}{l}\text { Hémangioblastome infratentoriel } \\
\text { Hémangioblastome médullaire } \\
\text { Hémangioblastome rétinien } \\
\text { Cancer du rein } \\
\text { Phéochromocytome } \\
\text { Kystes pancréatiques }\end{array}$ & $\begin{array}{c}9-68(29) \\
15-66(30) \\
0-68(25) \\
16-70(39) \\
5-58(27) \\
14-68(37)\end{array}$ & $\begin{array}{c}\text { IRM } \\
\text { IRM } \\
\text { FO } \\
\text { Écho/TDM } \\
\text { Métanéphrines//TDM } \\
\text { Écho/TDM }\end{array}$ & $\begin{array}{r}10 \text { ans } \\
10 \text { ans } \\
5 \text { ans } \\
15 \text { ans } \\
5 \text { ans } \\
15 \text { ans }\end{array}$ & $\begin{array}{l}1-3 \text { ans } \\
1-3 \text { ans } \\
\text { Biannuel } \\
\text { Biannuel } \\
\text { Annuel } \\
\text { Annuel }\end{array}$ & $\begin{array}{l}\text { 3-5 ans } \\
3-5 \text { ans } \\
\text { Annuel } \\
\text { Annuel } \\
\text { Annuel } \\
\text { Annuel }\end{array}$ & $\begin{array}{l}\text { Chirurgical si symptomatique } \\
\text { Chirurgical si symptomatique } \\
\text { Laser ou cryothérapie } \\
\text { Chirurgical } \\
\text { Chirurgical } \\
\text { Surveillance }\end{array}$ \\
\hline
\end{tabular}

D'après [3, 4, 6, 42, 43].

'Première valeur: âges extrêmes; seconde valeur: âge moyen.

2 FO: examen du fond d'cil. Angiographie en cas de doute.

${ }^{3}$ Scintigraphie à la méta-iodo-benzyl-guanidine (MIBG) en cas de suspicion. TDM = tomodensitométrie, IRM = imagerie par résonance magnétique.

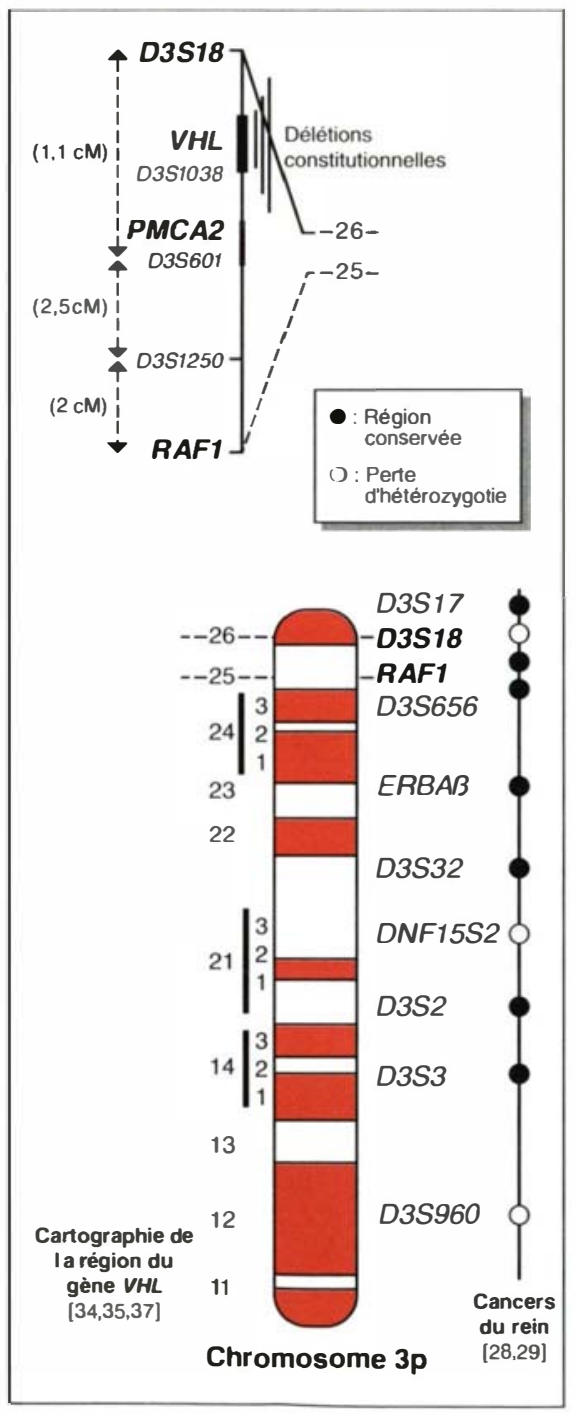

Figure 6. Représentation du bras court du chromosome 3 humain et de la région du gène VHL.
En l'absence de test fonctionnel, la relation de cause à effet, entre les variations observées au niveau de la séquence d'ADN et la présence clinique de la maladie de von Hippel Lindau, n'est cependant pas documentée. Enfin, aucune hypothèse n'est actuellement étayée concernant la réelle fonction du gène $V H I$.

\section{Diagnostic génétique}

Le diagnostic précoce de la maladie de VHL est maintenant possible par typage génétique. Son acquisition doit conduire à une modification progressive de la prise en charge des sujets à risque dans les familles atteintes, qui, jusqu'alors, devaient sans distinction se soumettre à une surveillance systématique contraignante. I a connaissance du gène responsable de la maladie de VHL et de la région chromosomique environnante permet de conduire l'analyse génétique, dans les familles atteintes, à partir de n'importe quel type cellulaire, l'altération du gène étant présente à l'état constitutionnel.

\section{Étude familiale par typage génétique}

Cette approche n'est envisageable que s'il existe des arguments cliniques formels en faveur d'une maladie de VHL, chez au moins un parent au premier degré de la personne dont on cherche à estimer le risque. La réalisation de l'étude dépend tout d'abord de la structure familiale : cette méthode est comparative et des in- formations cliniques concernant les proches apparentés des sujets à risque sont nécessaires, ainsi que leur prélèvement sanguin. En revanche, aucune information sur les sujets à risque n'est indispensable. Sa précision dépend ensuite du nombre de loci polymorphes étudiés, de leur informativité, de leur position par rapport au gène, et de la distance génétique qui les en sépare. L'utilisation de polymorphismes de type "microsatellite", très proches du gène, maintenant disponibles, rend le risque de recombinaison méiotique très faible, et assure une grande fiabilité à cette approche [42, 43]. I.eur autre intérêt est la possibilité de les révéler par des techniques d'amplification de type PC.R, ce qui diminue considérablement la quantité de matériel cellulaire nécessaire à l'étude ainsi que les délais expérimentaux. Cette méthode est actuellement disponible dans un but diagnostique à l'Institut Curie.

Diagnostic direct par recherche de mutation

La recherche directe de la mutation causale sur le gène $V H I$, chez un sujet à risque, lorsque cette dernière est connue dans une famille, fournit une information absolue et affranchit de l'étude familiale. La recherche systématique des mutations constitutionnelles devrait permettre à cette méthode de prendre progressivement le relais sur l'analyse par typage génétique. Il peut arriver que le diagnos- 


\section{RÉFÉRENCES}

35. Richards FM, Maher ER, Latif F, et al. Detailed genetic mapping of the von Hippel-Lindau disease tumour suppressor gene. $\int$ Med Genet $1993 ; 30: 104-7$.

36. Pericak-Vance MA, Nunes KJ, Whisenant $\mathrm{E}$, et al. Genetic mapping of dinucleotide repeat polymorphisms and von Hippel-Lindau disease on chromosome 3p25-p26. J Med Genet 1993; 30:487-91.

37. Vance JM, Small KW, Jones MA, et al. Confirmation of linkage in von Hippel-Lindau disease. Genomics $1990 ; 6$ : 565-7.

38. Latif F, Tory K, Gnarra J, et al. Identification of the von Hippel-Lindau disease tumor suppressor gene. Science 1993; 260 : 1317-20.

39. Richards FM, Crossey PA, Phipps ME, Foster K, Latif F, Evans G, Sampson J, Larman MI, Zbar B, Affara NA, Ferguson-Smith MA, Maher ER. Detailed mapping of germline deletions of the von Hippel-Lindau disease tumour suppressor gene. $\mathrm{Hum} \mathrm{Mol} \mathrm{Ge}$ net $1994 ; 3$ : 595-8.

40. Gnarra JR, Tory K, Weng Y, Schmidt L, Wei MH, Li H, Latif F, Liu $\$$, Chen F, Duh FM, Lubensky I, Duan DR, Florence C, Pozzatti R, Walther MM, Bander NH, Grossman HB, Brauch H, Pomer S, Brooks JD, Isaacs WB, Lerman MI, Zbar B, Linehan WM. Mutations of the VHL tumour suppressor gene in renal cell carcinoma. Nature Genet 1994 ; $7: 85-9$.

41. Grossey PA, Richards FM, Foster K, el al. Identification of intragenic mutations in the von Hippel-Lindau disease tumour suppressor gene and correlation with disease phenotype. Hum Mol Genet 1994 ; 3 ; 1303-8.

42. Maher ER, Bentley E, Payne SI, et al. Presymptomatic diagnosis of von HippelLindau disease with flanking DNA markers. I Med Genet 1992 ; 29 : 902-5.

43. Glenn GM, Linehan WM, Hosoe S, et al. Screening for von Hippel-Lindau disease by DNA polymorphism analysis. JAMA 1992 ; 267: $1226-31$.

44. Choyke PL, Filling-Katz MR, Shawker $\mathrm{TH}$, et al. Von Hippel-Lindau disease: radiologic screening for visceral manifestations. Radiology $1990 ; 174: 815-20$.

45. Jennings AM, Smith C, Cole DR, et al. Von Hippel-Lindau disease in a large British family : clinicopathologic features and recommendations for screening and followup. $Q J$ Med $1988 ; 251: 233-49$.

46. Richard S, Beigelman C, Gerber S, et al. L'hémangioblastome existe-t-il en dehors de la maladie de von Hippel-Lindau ? Neurochirurgie 1994; $40: 145-54$.

47. Neumann HPH, Berger DP, Sigmund G, et al. Pheochromocytomas, multiple endocrine neoplasia type 2, and von Hippel-Lin- tic de maladie de VHI, soit établi chez un patient sans contexte familial apparent, et que l'examen clinique de ses parents se révèle normal. Il s'agit dans ce cas d'une nouvelle mutation. I a proportion de ces formes non héritées n'est pas actuellement connue. Dans un but diagnostique pour la descendance de tels malades, ou pour un conseil génétique ultérieur, une étude familiale par typage génétique, comparative, n'est pas envisageable. En revanche, l'identification de la mutation responsable sur le gène VHL prend un intérêt prépondérant.

\section{Prise en charge médicale}

Elle est capitale pour le dépistage, la surveillance et le traitement des patients. Nombreux sont les malades reconnus tardivement, qu'il s'agisse de sujets à risque non examinés ou de malades chez lesquels l'affection s'est révélée par une atteinte névraxique ou viscérale isolée.

\section{Le dépistage des lésions}

I.es explorations systématiques, indispensables, ne doivent pas faire négliger l'essentielle enquête génétique avec étude généalogique soigneuse. A l'examen clinique complet des sujets à risque s'ajoutent un certain nombre d'explorations complémentaires : dosage des métanéphrines urinaires, imagerie par résonance magnétique (IRM) encéphalique et médullaire, examen ophtalmologique approfondi (fond d'oil et/ou angiographie), échographie abdominale suivie d'une tomodensitométrie (TDM) abdominale au moindre doute et parfois d'une scintigraphie à la méta-iodo-benzyl-guanidine (MIBG) (Tableau l) $[4,10,44,45]$. I a recherche en première intention du phéochromocytome est justifiée par les multiples investigations diagnostiques ou interventions chirurgicales nécessaires qui comportent un risque de crise hypertensive paroxystique aux conséquences dramatiques [10]. Ces explorations, certes fastidieuses, doivent être débutées tôt dans la vie et répétées régulièrement pour surveiller l'évolution de lésions connues ou dépister l'apparition de nouvelles atteintes chez les malades et les sujets à risque (Tableau I). L’importance d'un test génétique fiable permettant de limiter les examens aux seuls porteurs du gène délétère est ainsi soulignée. Le rôle du médecin traitant est essentiel dans le suivi médical et psychologique et l'indispensable collaboration avec les différents spécialistes.

I.es examens s'adressent bien sûr aux patients déjà connus et aux sujets à risque (descendance, fratrie) mais devraient également concerner tout malade présentant une tumeur pouvant survenir dans le cadre de la maladie de VHI. L'hémangioblastome est ainsi fréquemment lié à la maladie de VHL, bien plus qu'il n'est classiquement admis: dans une étude récente, $47 \%$ des cas d'hémangioblastomes infratentoriels et $77 \%$ des localisations médullaires survenus chez des sujets de moins de 30 ans ont pu être rattachés à la maladie [46]

Dans le cas du phéochromocytome, Neumann et al. ont trouvé une association à la maladie de VHI dans $24 \%$ des cas d'une série de 130 phéochromocytomes "sporadiques" [47]. Le problème du cancer rénal est plus complexe en raison de sa grande fréquence dans la population générale ( $3 \%$ des tumeurs de l'adulte). Il ne semble pas opportun d'examiner systématiquement tous les patients porteurs d'une tumeur rénale mais, devant une atteinte bilatérale, inhabituelle dans le cancer du rein sporadique (moins de $2 \%$ des cas), il est impératif de demander des explorations complètes [12].

\section{Possibilités thérapeutiques et pronostic}

L.e traitement des hémangioblastomes du système nerveux central est chirurgical [12]. La multiplicité des tumeurs, certaines localisations inextirpables et la possibilité de récidives en font toute la gravité. L.es lésions rétiniennes sont traitées par laser ou cryothérapie avec de bons résultats si les lésions sont accessibles et vues précocement [10]. La découverte d'un phéochromocytome impose son exérèse chirurgicale [12]. Les cancers rénaux posent de difficiles problèmes. Une néphrectomie bilatérale est justifiée devant des tumeurs multiples, bilatérales et volumineuses [12]. Quand les tumeurs sont peu nombreuses ou de petite taille, une chirurgie partielle est envisageable, d'où le rôle capital du dépistage pré- 
coce [12]. Une surveillance régulière à distance est cependant indispensable pour dépister l'apparition de nouvelles lésions ou la croissance de foyers tumoraux méconnus lors de l'intervention. Bien que l'évolution du cancer rénal semble plus lente chez les patients atteints de la maladie de VHL que dans la population générale, la survenue de métastases en fait la principale cause de décès des patients $[3,4]$.

I a sévérité de la maladie et son pronostic sont fonction du nombre de lésions, que rien ne permet actuellement de prévoir, et de leur topographie mais apparaissent surtout liés à la précocité du dépistage et du traitement qui doivent permettre d'augmenter l'espérance et la qualité de vie des sujets atteints (âge moyen de décès actuel : 49 ans [3]). Une étroite collaboration entre cliniciens et généticiens moléculaires n'en apparaît que plus indispensable

\section{Summary}

Von Hippel-Lindau disease

Von Hippel-Lindau disease is a rare genetic disease, with an autosomal dominant mode of inheritance, characterised by the development of several tumours, such as hemangioblastoma of the central nervous system and retina, renal cysts or carcinoma, pheochromocytoma and pancreatic cysts or tumors. Age of onset is variable but tumours usually occur in the range of 18 to 30 years of age. Point prevalence is estimated at $1 / 38( \pm 2)$ $\mathrm{x} .10^{3}$. Severe prognosis is due to late age of diagnosis, which is delayed because of non systematic screening and frequent phenotypic heterogeneity. The gene responsible for the disease, called $V H I$, localised on the short arm of chromosome 3 , has been identified last year. Its characterisation lead to more accurate presymptomatic diagnosis by the knowledge of intragenic and very closely linked DNA markers. Isolation of the gene opens the way to the precise analysis of the genotype/phenotype relationship in families affected by the von Hippel-Lindau disease. 